\title{
HOLOMORPHIC EXTENSION OF SMOOTH CR-MAPPINGS BETWEEN REAL-ANALYTIC AND REAL-ALGEBRAIC CR-MANIFOLDS *
}

\author{
FRANCINE MEYLAN ${ }^{\dagger}$, NORDINE MIR ${ }^{\ddagger}$, AND DMITRI ZAITSEV $§$
}

1. Introduction and results. The classical Schwarz reflection principle states that a continuous map $f$ between real-analytic curves $M$ and $M^{\prime}$ in $\mathbb{C}$ that locally extends holomorphically to one side of $M$, extends also holomorphically to a neighborhood of $M$ in $\mathbb{C}$. It is well-known that the higher-dimensional analog of this statement for maps $f: M \rightarrow M^{\prime}$ between real-analytic CR-submanifolds $M \subset \mathbb{C}^{N}$ and $M^{\prime} \subset \mathbb{C}^{N^{\prime}}$ does not hold without additional assumptions (unless $M$ and $M^{\prime}$ are totally real). In this paper, we assume that $f$ is $\mathcal{C}^{\infty}$-smooth and that the target $M^{\prime}$ is real-algebraic, i.e. contained in a real-algebraic subset of the same dimension. If $f$ is known to be locally holomorphically extendible to one side of $M$ (when $M$ is a hypersurface) or to a wedge with edge $M$ (when $M$ is a generic submanifold of higher codimension), then $f$ automatically satisfies the tangential Cauchy-Riemann equations, i.e. it is CR. On the other hand, if $M$ is minimal, any CR-map $f: M \rightarrow M^{\prime}$ locally extends holomorphically to a wedge with edge $M$ by Tumanov's theorem [Tu88] and hence, in that case, the extension assumption can be replaced by assuming $f$ to be CR.

Local holomorphic extension of a CR-map $f: M \rightarrow M^{\prime}$ may clearly fail when $M^{\prime}$ contains an (irreducible) complex-analytic subvariety $E^{\prime}$ of positive dimension and $f(M) \subset E^{\prime}$. Indeed, any nonextendible CR-function on $M$ composed with a nontrivial holomorphic map from a disc in $\mathbb{C}$ into $E^{\prime}$ yields a counterexample. Our first result shows that this is essentially the only exception. Denote by $\mathcal{E}^{\prime}$ the set of all points $p^{\prime} \in M^{\prime}$ through which there exist irreducible complex-analytic subvarieties of $M^{\prime}$ of positive dimension. We prove:

THEOREM 1.1. Let $M \subset \mathbb{C}^{N}$ and $M^{\prime} \subset \mathbb{C}^{N^{\prime}}$ be respectively connected realanalytic and real-algebraic CR-submanifolds. Assume that $M$ is minimal at a point $p \in M$. Then for any $\mathcal{C}^{\infty}$-smooth CR-map $f: M \rightarrow M^{\prime}$, at least one of the following conditions holds:

(i) $f$ extends holomorphically to a neighborhood of $p$ in $\mathbb{C}^{N}$;

(ii) $f$ sends a neighborhood of $p$ in $M$ into $\mathcal{E}^{\prime}$.

If $M^{\prime}$ is a real-analytic hypersurface, the set $\mathcal{E}^{\prime}$ consists exactly of those points that are not of finite type in the sense of D'ANGELo [D'A82] (see LEMPERT [L86] for the proof) and, in particular, $\mathcal{E}^{\prime}$ is closed. The same fact also holds if $M^{\prime}$ is any realanalytic submanifold or even any real-analytic subvariety (see [D'A91]). However, in general, $\mathcal{E}^{\prime}$ may not even be a real-analytic subset (see Example 2.1). In case $\mathcal{E}^{\prime}=V^{\prime}$ is a subvariety, we have:

Corollary 1.2. Let $M \subset \mathbb{C}^{N}$ and $M^{\prime} \subset \mathbb{C}^{N^{\prime}}$ be as in Theorem 1.1. Assume that $M$ is minimal at a point $p \in M$ and that all positive-dimensional irreducible complexanalytic subvarieties in $M^{\prime}$ are contained in a fixed (complex-analytic) subvariety $V^{\prime} \subset$

\footnotetext{
*Received December 10, 2002; accepted for publication June 6, 2003.

†Institut de Mathématiques, Université de Fribourg, 1700 Perolles, Fribourg, Switzerland (francine.meylan@unifr.ch).

‡Université de Rouen, Laboratoire de Mathématiques Raphaël Salem, UMR 6085 CNRS, 76821 Mont-Saint-Aignan Cedex, France (Nordine.Mir@univ-rouen.fr).

$\S$ School of Mathematics, Trinity College, Dublin 2, Ireland (zaitsev@maths.tcd.ie).
} 
$M^{\prime}$. Then any $\mathcal{C}^{\infty}$-smooth $C R$-map $f: M \rightarrow M^{\prime}$ that does not send a neighborhood of $p$ in $M$ into $V^{\prime}$ extends holomorphically to a neighborhood of $p$ in $\mathbb{C}^{N}$.

In view of an example due to EBENFELT [E96], the minimality assumption on $M$ at $p$ in Corollary 1.2 cannot be replaced by the assumption that $M$ is minimal somewhere. On the other hand, if $M$ is also real-algebraic, this replacement is possible:

THEOREM 1.3. Let $M \subset \mathbb{C}^{N}$ and $M^{\prime} \subset \mathbb{C}^{N^{\prime}}$ be connected real-algebraic $C R$ submanifolds with $p \in M$ and let $V^{\prime} \subset M^{\prime}$ be as in Corollary 1.2. Then the conclusion of Corollary 1.2 holds provided $M$ is minimal somewhere.

In the setting of Theorem 1.3, any $\mathcal{C}^{\infty}$-smooth CR-map $f: M \rightarrow M^{\prime}$ that does not send a neighborhood of $p$ in $M$ into $V^{\prime}$ extends even algebraically to a neighborhood of $p$ in $\mathbb{C}^{N}$ by a result of [Z99] (see $\S 7$ ). Since the subset $\mathcal{E}^{\prime} \subset M^{\prime}$ is always closed, Corollary 1.2 and Theorem 1.3 imply:

Corollary 1.4. Let $M \subset \mathbb{C}^{N}$ and $M^{\prime} \subset \mathbb{C}^{N^{\prime}}$ be respectively connected realanalytic and real-algebraic $C R$-submanifolds. Assume that $M$ is minimal at a point $p \in M$ and that $M^{\prime}$ does not contain any irreducible complex-analytic subvariety of positive dimension through a point $p^{\prime} \in M^{\prime}$. Then any $\mathcal{C}^{\infty}$-smooth $C R$-map $f: M \rightarrow$ $M^{\prime}$ with $f(p)=p^{\prime}$ extends holomorphically to a neighborhood of $p$ in $\mathbb{C}^{N}$. The same conclusion holds at a point $p \in M$ if $M$ is real-algebraic and only somewhere minimal.

In the case when $M \subset \mathbb{C}^{N}$ is a real hypersurface, the first part of Corollary 1.4 is due to Pushnikov [P90a, P90b] (see also [CPS00]).

A prototype of a target real-algebraic $\mathrm{CR}$-submanifold with no nontrivial complexanalytic subvariety is given by the unit sphere $\mathbb{S}^{2 N^{\prime}-1} \subset \mathbb{C}^{N^{\prime}}$. Even in that case, Corollary 1.4 seems to be new. Indeed, we have:

Corollary 1.5. Let $M \subset \mathbb{C}^{N}$ be a connected real-analytic CR-submanifold, minimal at a point $p \in M$. Then any $\mathcal{C}^{\infty}$-smooth $C R$-map $f: M \rightarrow \mathbb{S}^{2 N^{\prime}-1}$ extends holomorphically to a neighborhood of $p$ in $\mathbb{C}^{N}$. The same conclusion holds for any point $p \in M$ if $M$ is real-algebraic and only somewhere minimal.

For $f$ of class $\mathcal{C}^{\infty}$, Corollary 1.5 extends results of WEBSTER [W79], Forstnerič [F86, F89, F92], HuANG [H94] and BAOUENDI-HuANG-Rothschild [BHR96]. (On the other hand, in their setting, they prove holomorphic extension of $f$ of $\operatorname{class} \mathcal{C}^{k}$ for appropriate $k$.)

If we restrict ourselves to submersive CR-maps (i.e. maps for which the differential is surjective), a known obstruction to their holomorphic extension is the holomorphic degeneracy of the submanifolds. Recall that a real-analytic CR-submanifold $M$ is holomorphically degenerate (see STANTON [S96]) at a point $p \in M$ if there is a nontrivial holomorphic vector field in a neighborhood of $p$ in $\mathbb{C}^{N}$ whose real and imaginary parts are tangent to $M$. The existence of such a vector field and a nonextendible CR-function on $M$ at $p$ yields nonextendible local self CR-diffeomorphic maps of $M$ near $p$ (see [BHR96]). It is known (see [BER96]) that $M$ is holomorphically degenerate at $p$ if and only if it is holomorphically degenerate everywhere on the connected component of $p$. Our next result shows that for source minimal CR-submanifolds, holomorphic degeneracy is essentially the only obstruction for submersive CR-maps to be holomorphically extendible.

THEOREM 1.6. Let $M \subset \mathbb{C}^{N}$ and $M^{\prime} \subset \mathbb{C}^{N^{\prime}}$ be respectively connected realanalytic and real-algebraic $C R$-submanifolds of the same $C R$-codimension with $p \in M$. Assume that $M$ is everywhere minimal and $M^{\prime}$ is holomorphically nondegenerate. 
Then any $\mathcal{C}^{\infty}$-smooth $C R$-map $f: M \rightarrow M^{\prime}$ which is somewhere submersive extends holomorphically to a neighborhood of $p$ in $\mathbb{C}^{N}$.

In the case when $M, M^{\prime} \subset \mathbb{C}^{N}$ are real hypersurfaces, a similar result is contained in [CPS00]. Example 2.2 below shows that the assumption that $M$ is everywhere minimal cannot be replaced in Theorem 1.6 by the weaker assumption that $M$ is minimal at $p$. On the other hand, if $M$ is real-algebraic, a replacement with even weaker assumption on $M$ is possible:

THEOREM 1.7. Let $M \subset \mathbb{C}^{N}$ and $M^{\prime} \subset \mathbb{C}^{N^{\prime}}$ be connected real-algebraic $C R$ submanifolds of the same CR-codimension with $p \in M$. Then the conclusion of Theorem 1.6 holds provided $M$ is somewhere minimal and $M^{\prime}$ is holomorphically nondegenerate.

In the setting of Theorem 1.7, any $\mathcal{C}^{\infty}$-smooth CR-map $f: M \rightarrow M^{\prime}$ extends in fact algebraically to a neighborhood of $p$ in $\mathbb{C}^{N}$ by a result of [Z99] (see $\S 7$ ). Theorem 1.7 extends a result of [BHR96] who obtained the same conclusion for $M, M^{\prime} \subset \mathbb{C}^{N}$ real-algebraic hypersurfaces and of KoJCINOvic [K00] for $M, M^{\prime} \subset \mathbb{C}^{N}$ generic submanifolds of equal dimension. For further related results and history on the analyticity problem for CR-mappings, the reader is referred to [F93, BER99, H01].

We shall derive the above results in $\S 7$ from the following statement that relates analyticity properties of a smooth CR-map with geometric properties of its graph:

THEOREM 1.8. Let $M \subset \mathbb{C}^{N}$ and $M^{\prime} \subset \mathbb{C}^{N^{\prime}}$ be respectively connected realanalytic and real-algebraic CR-submanifolds and $f: M \rightarrow M^{\prime}$ a $\mathcal{C}^{\infty}$-smooth $C R$-map whose graph is denoted by $\Gamma_{f}$. Assume that $M$ is minimal at a point $p \in M$ and that $f$ does not extend holomorphically to any neighborhood of $p$. Then there exists an integer $1 \leq n \leq N^{\prime}-1$ and a real-analytic subset $A \subset M \times M^{\prime}$ through $(p, f(p))$ containing a neighborhood $\Omega$ of $(p, f(p))$ in $\Gamma_{f}$ and satisfying the following straightening property: for any point $(q, f(q))$ in a dense open subset of $\Omega$, there exists a neighborhood $U_{q}$ of $(q, f(q))$ in $\mathbb{C}^{N} \times \mathbb{C}^{N^{\prime}}$ and a holomorphic change of coordinates in $U_{q}$ of the form $\left(\tilde{z}, \tilde{z}^{\prime}\right)=\left(z, \varphi\left(z, z^{\prime}\right)\right) \in \mathbb{C}^{N} \times \mathbb{C}^{N^{\prime}}$ such that

$$
A \cap U_{q}=\left\{\left(z, z^{\prime}\right) \in U_{q}: z \in M, \tilde{z}_{n+1}^{\prime}=\cdots=\tilde{z}_{N^{\prime}}^{\prime}=0\right\} .
$$

Theorem 1.8 will follow from the more general Theorem 6.1, where the target $M^{\prime} \subset \mathbb{C}^{N^{\prime}}$ is assumed to be a real-algebraic subset and an estimate for the number $n$ (in Theorem 1.8) is given. Our approach follows partially the techniques initiated in [P90a, P90b] and followed in [CPS00] in the case $M$ is a hypersurface. A crucial point in the proof of Theorem 1.8 consists of showing (after possible shrinking $M$ around $p$ ) that near a generic point of the graph $\Gamma_{f}$, the intersection of $M \times \mathbb{C}^{N^{\prime}}$ with the local Zariski closure of $\Gamma_{f}$ at $(p, f(p))$ (see $\S 4$ for the definition) is contained in $M \times M^{\prime}$ (see Theorem 6.1 and Proposition 6.2). Here we have to proceed differently from [P90a, $\mathrm{P} 90 \mathrm{~b}, \mathrm{CPS} 00]$. In $\S 3$ we give preliminary results based on a meromorphic extension property from [MMZ02]. In particular, Proposition 3.4 (ii) may be of independent interest. $\S 4-6$ are devoted to the proof of Theorem 1.8 .

REMARK 1.9. A preprint version of this paper was posted on the web on January, 28, 2002 (http://www.arxiv.org/abs/math.CV/0201267). Later the authors discovered the note by Coupet, Damour, Merker and Sukhov, "Sur l'analyticité des applications CR lisses à valeurs dans un ensemble algébrique réel", C. R. Math. Acad. Sci. Paris, 334 (2002), no. 11, 953-956 (submitted on March 12, 2002), containing results and 
(sketched) proofs analogous to those in the posted preprint that were not contained in previous literature (in particular, the arguments of $\S 3$ there deviate from those in [CPSO0] but follow $\S 3$ and $\S 6$ of this paper).

Acknowledgment. The authors would like to thank M.S. BAOUENDI, P. EBENFELT, H.-M. MAIRE and L.P. RothsCHILD for their interest to the paper and many helpful discussions.

\section{Preliminaries and examples.}

2.1. CR-submanifolds and CR-maps. A real submanifold $M \subset \mathbb{C}^{N}$ is called a CR-submanifold if the dimension of the complex tangent space $T_{p}^{c} M:=T_{p} M \cap i T_{p} M$ is independent of $p \in M$. In this case $\operatorname{dim}_{\mathbb{C}} T_{p}^{c} M$ is called the $C R$-dimension and $\operatorname{dim}_{\mathbb{R}} T_{p} M-\operatorname{dim}_{\mathbb{R}} T_{p}^{c} M$ the $C R$-codimension of $M$. Furthermore, $M$ is called generic if for any point $p \in M$, one has $T_{p} M+i T_{p} M=T_{p} \mathbb{C}^{N}$. For a CR-submanifold $M$ we write $T^{0,1} M:=T^{0,1} \mathbb{C}^{N} \cap \mathbb{C} T M$, where $T^{0,1} \mathbb{C}^{N}$ is the bundle of $(0,1)$ tangent vectors in $\mathbb{C}^{N}$. A function $h: M \rightarrow \mathbb{C}^{N^{\prime}}$ of class $\mathcal{C}^{1}$ is called a CR-function if for any section $L$ of the CR-bundle, $L f=0$. If $h$ is merely continuous, $h$ is still called CR if it is annihilated by all vector fields $L$ as above in the sense of distributions. A continuous map $f: M \rightarrow M^{\prime}$ between CR-submanifolds $M \subset \mathbb{C}^{N}$ and $M^{\prime} \subset \mathbb{C}^{N^{\prime}}$ is called a CR-map if all its components are CR-functions.

A CR-submanifold $M \subset \mathbb{C}^{N}$ is called minimal (in the sense of TumANov) at a point $p \in M$ if there is no real submanifold $S \subset M$ through $p$ with $\operatorname{dim} S<\operatorname{dim} M$ and such that $T_{q}^{c} M \subset T_{q} S$, for all $q \in S$. It is well-known that if $M$ is real-analytic, the minimality condition of $M$ is equivalent to the finite type condition in the sense of KoHN and BLOOM-GRAHAM (see [BER99]).

A real (resp. complex) submanifold $M \subset \mathbb{C}^{N}$ is real-algebraic (resp. algebraic) if it is contained in a real-algebraic (resp. complex-algebraic) subvariety with the same real (resp. complex) dimension as that of $M$. A map $f: M \rightarrow M^{\prime}$ between real submanifolds $M \subset \mathbb{C}^{N}$ and $M^{\prime} \subset \mathbb{C}^{N^{\prime}}$ is real-algebraic if its graph $\Gamma_{f}:=\{(z, f(z))$ : $z \in M\}$ is a real-algebraic submanifold of $\mathbb{C}^{N} \times \mathbb{C}^{N^{\prime}}$. Similarly, a holomorphic map between open subsets $\Omega \subset \mathbb{C}^{N}$ and $\Omega^{\prime} \subset \mathbb{C}^{N^{\prime}}$ is called algebraic if its graph is a complex-algebraic submanifold of $\Omega \times \Omega^{\prime}$.

2.2. Examples. The following example shows that, even if $M^{\prime} \subset \mathbb{C}^{N^{\prime}}$ is a realanalytic hypersurface, the subset $\mathcal{E}^{\prime} \subset M^{\prime}$ of all points that are not of finite D'Angelo type is not real-analytic in general.

EXAmple 2.1. Consider the tube real-analytic hypersurface $M^{\prime} \subset \mathbb{C}^{4}$ given by

$$
\left(\operatorname{Re} z_{1}\right)^{2}-\left(\operatorname{Re} z_{2}\right)^{2}+\left(\operatorname{Re} z_{3}\right)^{2}=\left(\operatorname{Re} z_{4}\right)^{3}
$$

near the point $(1,1,0,0) \in M^{\prime}$. We claim that the subset $\mathcal{E}^{\prime} \subset M^{\prime}$ is given by $\operatorname{Re} z_{4} \geq 0$ and is therefore not analytic. Indeed, every intersection of $M^{\prime}$ with $\left\{z_{4}=\right.$ const, $\left.\operatorname{Re} z_{4} \geq 0\right\}$ contains complex lines through each point and is hence everywhere of D'ANGELO infinite type. On the other hand, if $\operatorname{Re} z_{4}<0$, the coordinate $\operatorname{Re} z_{2}$ can be expressed as a strictly convex function of the other coordinates. Therefore, $M^{\prime}$ is strictly pseudoconvex at each such point and thus of D'ANGELO finite type.

The following example shows that a somewhere submersive $\mathcal{C}^{\infty}$-smooth CR-map $f: M \rightarrow M^{\prime}$ between connected real-analytic hypersurfaces in $\mathbb{C}^{2}$ can be real-analytic on some connected component of the set of minimal points of $M$ and not real-analytic 
in another component. In particular, the assumption of Theorem 1.6 that $M$ is everywhere minimal cannot be replaced by the weaker assumption that $M$ is minimal at $p$.

EXAMPLe 2.2. As in EBENFELT's example [E96], let $M, M^{\prime} \subset \mathbb{C}^{2}$ be connected real-analytic hypersurfaces through 0 given respectively by

$$
\operatorname{Im} w=\theta\left(\arctan |z|^{2}, \operatorname{Re} w\right), \quad \operatorname{Im} w=(\operatorname{Re} w)|z|^{2},
$$

where $t=\theta(\xi, s)$ is the unique solution of the algebraic equation $\xi\left(t^{2}+s^{2}\right)-t=0$ with $\theta(0,0)=0$ given by the implicit function theorem. Note that $M$ and $M^{\prime}$ are minimal precisely outside the complex line $\{w=0\}$ and that $M^{\prime}$ is real-algebraic, but $M$ is not. For every $\mathcal{C}^{\infty}$-smooth $C R$-function $\varphi$ on $M$, define a map $f_{\varphi}: M \rightarrow \mathbb{C}^{2}$ by

$$
f_{\varphi}(z, w):= \begin{cases}(z, 0) & \operatorname{Re} w=0 \\ \left(z, e^{-1 / w}\right) & \operatorname{Re} w>0 \\ \left(z+\varphi(z, w) e^{1 / w}, 0\right) & \operatorname{Re} w<0\end{cases}
$$

By similar arguments as in [E96] it follows that $f_{\varphi}$ is always a $\mathcal{C}^{\infty}$-smooth CR-map sending $M$ into $M^{\prime}$. Suppose we can choose $\varphi$ not holomorphically extendible to any neighborhood in $\mathbb{C}^{2}$ of a fixed minimal point $p_{0}=\left(z_{0}, w_{0}\right) \in M$ with $\operatorname{Re} w_{0}<0$. Then it is easy to see that $f_{\varphi}$ is somewhere submersive but does not extend holomorphically to any neighborhood of the minimal point $p_{0} \in M$.

To show that the above choice of $p_{0}$ and $\varphi$ is possible, observe that $\theta$ can be factored as $\theta(\xi, s)=s^{2} \xi(1+\widetilde{\theta}(\xi, s))$ with $\widetilde{\theta}$ analytic and vanishing at the origin. Hence $\operatorname{Im} w \geq 0$ for every sufficiently small $(z, w) \in M$. Then, for any real sufficiently small $x_{0} \neq 0$, the point $p_{0}:=\left(0, x_{0}\right) \in M$ is minimal and a suitable branch of $e^{-1 /\left(w-x_{0}\right)^{1 / 3}}$ extends to a $\mathcal{C}^{\infty}$-smooth $C R$-function $\varphi$ on $M$ that is not holomorphically extendible to any neighborhood of $p_{0}$.

3. A result on meromorphic extension and its applications. In what follows, for any subset $V \subset \mathbb{C}^{k}, V^{*}$ denotes the set $\{\bar{z}: z \in V\}$ and, as usual, for any ring $A$, we denote by $A[X], X=\left(X_{1}, \ldots, X_{s}\right)$, the ring of polynomials in $s$ indeterminates with coefficients in $A$. An important role in the proof of Theorem 1.8 will be played by the following meromorphic extension result from [MMZ02, Theorem $2.6]$.

THEOREM 3.1. Let $\Omega \subset \mathbb{C}^{N}, V \subset \mathbb{C}^{k}$ be open subsets, $M \subset \Omega$ a connected generic real-analytic submanifold, $G: M \rightarrow V$ a continuous $C R$-function and $\Phi, \Psi: V^{*} \times \Omega \rightarrow$ $\mathbb{C}$ holomorphic functions. Assume that $M$ is minimal at every point and that there exists a nonempty open subset of $M$ where $\Psi(\overline{G(z)}, z)$ does not vanish and where the quotient

$$
H(z):=\frac{\Phi(\overline{G(z)}, z)}{\Psi(\overline{G(z)}, z)}
$$

is CR. Then $\Psi(\overline{G(z)}, z)$ does not vanish on a dense open subset $\widetilde{M} \subset M$ and $H$ extends from $\widetilde{M}$ meromorphically to a neighborhood of $M$ in $\mathbb{C}^{N}$.

REMARK 3.2. Results in the spirit of Theorem 3.1 have been important steps in proving regularity results for CR-mappings (see e.g. [P90a, P90b, BHR96, CPS99, 
CPS00, MMZ02, Mi03]). Also a special case of Theorem 3.1 with $G$ being $\mathcal{C}^{\infty}$ and $H$ $C R$ on a dense open subset is due to [Da01] but the stronger form of Theorem 3.1 is essential to complete the proofs in this paper and does not follow from the arguments of [Da01].

For a generic real-analytic submanifold $M \subset \mathbb{C}^{N}$, denote by $\mathcal{C}^{\infty}(M)$ the ring of $\mathcal{C}^{\infty}$-smooth functions on $M$, by $\mathcal{O}(M)$ the ring of restrictions of holomorphic functions to $M$ and by $\mathcal{O}_{p}(M)$ the corresponding ring of germs at a point $p \in M$. Similarly to [CPS99] (see also [P90a, P90b, CPS00, MMZ02]), define a subring $\mathcal{A}(M) \subset \mathcal{C}^{\infty}(M)$ as follows: a function $\eta \in \mathcal{C}^{\infty}(M)$ belongs to $\mathcal{A}(M)$ if and only if, near every point $p \in M$, it can be written in the form $\eta(z) \equiv \Phi(\overline{G(z)}, z)$, where $G$ is a $\mathbb{C}^{k}$-valued $\mathcal{C}^{\infty}$. smooth $C R$-function in a neighborhood of $p$ in $M$ for some $k$ and $\Phi$ is a holomorphic function in a neighborhood of $(\overline{G(p)}, p)$ in $\mathbb{C}^{k} \times \mathbb{C}^{N}$. Note that the ring $\mathcal{C}^{\omega}(M)$ of all real-analytic functions on $M$ is a subring of $\mathcal{A}(M)$. We have the following known properties (see e.g. [MMZ02]):

LemMA 3.3. Let $M \subset \mathbb{C}^{N}$ be a connected generic real-analytic submanifold that is minimal at every point. Then for any $u \in \mathcal{A}(M)$ the following hold:

(i) if $u$ vanishes on a nonempty open subset of $M$, then it vanishes identically on $M$;

(ii) if $L$ is a real-analytic $(0,1)$ vector field on $M$, then $L u \in \mathcal{A}(M)$.

The following proposition is a consequence of Theorem 3.1 and will be essential for the proof of Theorem 1.8. In the proof we follow the approach of [P90b] (see also [CMS99, Proposition 5.1]).

Proposition 3.4. Let $M \subset \mathbb{C}^{N}$ be a connected generic real-analytic submanifold that is minimal at every point. Let $F_{1}, \ldots, F_{r}$ be $\mathcal{C}^{\infty}$-smooth $C R$-functions on $M$ satisfying one of the following conditions:

(i) the restrictions of $F_{1}, \ldots, F_{r}$ to a nonempty open subset of $M$ satisfy a nontrivial polynomial identity with coefficients in $\mathcal{A}(M)$;

(ii) the restrictions of $F_{1}, \ldots, F_{r}, \overline{F_{1}}, \ldots, \overline{F_{r}}$ to a nonempty open subset of $M$ satisfy a nontrivial polynomial identity with coefficients in $\mathcal{C}^{\omega}(M)$.

Then for any point $q \in M$, the germs at $q$ of $F_{1}, \ldots, F_{r}$ satisfy a nontrivial polynomial identity with coefficients in $\mathcal{O}_{q}(M)$.

Proof. We first observe that, for the rest of the proof, we can assume that the $(0,1)$ vector fields on $M$ are spanned by global real-analytic vector fields on $M$. Indeed, suppose we have proved Proposition 3.4 under this additional assumption, then we claim that Proposition 3.4 follows from that case. For this, for fixed $F_{1}, \ldots, F_{r}$ as in Proposition 3.4 (i) (or (ii)), let $\Omega \subset M$ be the set of all points $q \in M$ for which the conclusion holds. Then $\Omega$ is clearly open. After shrinking $M$ appropriately, we see that $\Omega \neq \emptyset$ by the above weaker supposed version of Proposition 3.4. Analogously, shrinking $M$ around an accumulation point of $\Omega$, we conclude that $\Omega$ is closed and therefore $\Omega=M$ as required.

Let now $\mathcal{R}(T)$ be a nontrivial polynomial in $T=\left(T_{1}, \ldots, T_{r}\right)$ over $\mathcal{A}(M)$ such that

$$
\left.\mathcal{R}(F)\right|_{U} \equiv 0
$$

for some nonempty open subset $U \subset M$, where $F:=\left(F_{1}, \ldots, F_{r}\right)$. We write $\mathcal{R}(T)$ as 
a linear combination

$$
\mathcal{R}(T)=\sum_{j=1}^{l} \delta_{j} r_{j}(T),
$$

where each $\delta_{j} \neq 0$ is in $\mathcal{A}(M)$ and $r_{j}$ is a monomial in $T$. By Lemma 3.3, each $\delta_{j}$ does not vanish on a dense open subset of $M$. By shrinking $U$, we may assume that $\delta_{l}$ does not vanish at every point of $U$. We prove the desired conclusion by induction on the number $l$ of monomials in (3.2). For $l=1,(3.1)$ and (3.2) and the choice of $U$ imply that $\left.r_{1}(F)\right|_{U}=0$. Since $r_{1}$ is a monomial and each component of $\bar{F}$ is in $\mathcal{A}(M)$, it follows from Lemma 3.3 that $F_{j}=0$ for some $j$ which yields the required nontrivial polynomial identity with coefficients in $\mathcal{O}(M)$ (even in $\mathbb{C}$ ).

Suppose now that the desired conclusion holds for any polynomial $\mathcal{R}$ whose number of monomials is strictly less than $l$. In view of (3.1) and (3.2) we have

$$
\left.r_{l}(F)\right|_{U}+\left.\left(\sum_{j<l} \frac{\delta_{j}}{\delta_{l}} r_{j}(F)\right)\right|_{U}=0 .
$$

Let $L$ be any global CR vector field on $M$ with real-analytic coefficients. Applying $L$ to (3.3) and using the assumption that $F_{j}$ is CR for any $j$, we obtain

$$
\left.\left(\sum_{j<l} L\left(\frac{\delta_{j}}{\delta_{l}}\right) r_{j}(F)\right)\right|_{U}=0 .
$$

By Lemma 3.3 (ii), each coefficient $L\left(\delta_{j} / \delta_{l}\right)$ can be written as a ratio of two functions in $\mathcal{A}(M)$. From (3.4), we are led to distinguish two cases. If for some $j \in\{1, \ldots, l-1\}$, $L\left(\delta_{j} / \delta_{l}\right)$ does not vanish identically in $U$, then the required conclusion follows from the induction hypothesis.

It remains to consider the case when

$$
L\left(\delta_{j} / \delta_{l}\right)=0, \quad \text { in } U,
$$

for all $j$ and for all choices of $(0,1)$ vector field $L$. Then (3.5) implies that each ratio $\delta_{j} / \delta_{l}$ is CR on $U$ by the assumption at the beginning of the proof. Hence, by Theorem 3.1 , it follows that each $\delta_{j} / \delta_{l}$ extends meromorphically to a neighborhood of $M$ in $\mathbb{C}^{N}$ and therefore, (3.3) can be rewritten as

$$
\left.r_{l}(F)\right|_{U}+\left.\left(\sum_{j<l} m_{j} r_{j}(F)\right)\right|_{U}=0
$$

with $m_{1}, \ldots, m_{l-1}$ being restrictions to $M$ of meromorphic functions in a neighborhood of $M$. Since $M$ is connected and minimal everywhere, the identity

$$
r_{l}(F(z))+\sum_{j<l} m_{j}(z) r_{j}(F(z))=0
$$

holds for every $z \in M$ outside the set $S$ consisting of the poles of the $m_{j}$ 's. This proves the desired conclusion under the assumption (i).

For the statement under the assumption (ii), consider a nontrivial polynomial $\mathcal{P}(T, \widetilde{T}) \in \mathcal{C}^{\omega}(M)[T, \widetilde{T}]$ such that $\left.\mathcal{P}(F, \bar{F})\right|_{U}=0$ for a non-empty open subset $U \subset M$. We write

$$
\mathcal{P}(T, \widetilde{T})=\sum_{\nu \in \mathbb{N}^{r},|\nu| \leq l} \mathcal{P}_{\nu}(\widetilde{T}) T^{\nu}
$$


where each $\mathcal{P}_{\nu}(\widetilde{T}) \in \mathcal{C}^{\omega}(M)[\widetilde{T}]$ and at least one of the $\mathcal{P}_{\nu}$ 's is nontrivial. If there exists $\nu_{0} \in \mathbb{N}^{r}$ such that $\mathcal{P}_{\nu_{0}}(\bar{F})$ is not zero in the ring $\mathcal{A}(M)$, then it follows that the polynomial $\mathcal{Q}(T):=\mathcal{P}(T, \bar{F}) \in \mathcal{A}(M)[T]$ is nontrivial and satisfies $\left.\mathcal{Q}(F)\right|_{U}=0$. Then the condition (i) is fulfilled and the required conclusion is proved above.

It remains to consider the case when $\mathcal{P}_{\nu}(\bar{F})=0$ for any $\nu \in \mathbb{N}^{r}$. Fix any $\nu$ such that $\mathcal{P}_{\nu}(\widetilde{T})$ is nontrivial. Let $\overline{\mathcal{P}_{\nu}}(T)$ denote the polynomial in $\mathcal{C}^{\omega}(M)[T]$ obtained from $\mathcal{P}_{\nu}$ by taking the complex conjugates of its coefficients. Then $\overline{\mathcal{P}_{\nu}}(T)$ is a nontrivial polynomial in $\mathcal{A}(M)[T]$ and satisfies $\overline{\mathcal{P}_{\nu}}(F)=0$ on $M$. Here again, condition (i) is fulfilled and the desired conclusion follows. The proof is complete.

4. Zariski closure of the graph of a CR-map. Throughout this section, let $M \subset \mathbb{C}^{N}$ be a real-analytic generic submanifold, $p \in M$ a fixed point in $M$ and $f: M \rightarrow \mathbb{C}^{N^{\prime}}$ a $\mathcal{C}^{\infty}$-smooth CR-map. For $q \in \mathbb{C}^{N}$, denote by $\mathcal{O}_{q}$ the ring of germs at $q$ of holomorphic functions in $\mathbb{C}^{N}$. The goal of this section is to define and give some basic properties of the local Zariski closure of the graph $\Gamma_{f}$ at $(p, f(p))$ over the ring $\mathcal{O}_{p}\left[z^{\prime}\right]$.

4.1. Definition of the local Zariski closure. For $M, f$ and $p$ as above, define the (local) Zariski closure of $\Gamma_{f}$ at $(p, f(p))$ with respect to the ring $\mathcal{O}_{p}\left[z^{\prime}\right]$ as the germ $\mathcal{Z}_{f} \subset \mathbb{C}^{N} \times \mathbb{C}^{N^{\prime}}$ at $(p, f(p))$ of a complex-analytic set defined by the zero-set of all elements in $\mathcal{O}_{p}\left[z^{\prime}\right]$ vanishing on $\Gamma_{f}$. Note that since $\mathcal{Z}_{f}$ contains the germ of the graph of $f$ through $(p, f(p))$, it follows that $\operatorname{dim}_{\mathbb{C}} \mathcal{Z}_{f} \geq N$. In what follows, we shall denote by $\mu_{p}(f)$ the dimension of the Zariski closure $\mathcal{Z}_{f}$.

REMARK 4.1. Observe that if $M$ is furthermore assumed to be minimal at $p$, all the components of the map $f$ extend to a wedge with edge $M$ at $p$; in this case, it follows from unique continuation at the edge that $\mathcal{Z}_{f}$ is locally irreducible with respect to the ring $\mathcal{O}_{p}\left[z^{\prime}\right]$.

4.2. Dimension of the local Zariski closure and transcendence degree. In this section, we discuss a link between the dimension of the Zariski closure $\mu_{p}(f)$ defined above and the notion of transcendence degree considered in [P90a, P90b, CMS99, CPS00]. The reader is referred to [ZS58] for basic notions from field theory used here.

Since the ring $\mathcal{O}_{p}(M)$ is an integral domain, one may consider its quotient field that we denote by $\mathcal{M}_{p}(M)$. Recall that, by a theorem of TomAssini [To66], any germ in $\mathcal{O}_{p}(M)$ extends holomorphically to a neighborhood of $p$ in $\mathbb{C}^{N}$. Hence an element belongs to $\mathcal{M}_{p}(M)$ if and only if it extends meromorphically to a neighborhood of $p$ in $\mathbb{C}^{N}$. Note that if $M$ is moreover assumed to be minimal at $p$, it follows that the ring of germs at $p$ of $\mathcal{C}^{\infty}$-smooth CR-functions on $M$ is an integral domain, which allows one to introduce its quotient field containing $\mathcal{M}_{p}(M)$. Therefore, for a generic submanifold $M$ minimal at $p$, one may consider the finitely generated field extension $\mathcal{M}_{p}(M)\left(f_{1}, \ldots, f_{N^{\prime}}\right)$ over $\mathcal{M}_{p}(M)$ where $f_{1}, \ldots, f_{N^{\prime}}$ are the components of $f$ considered as germs at $p$. (In the hypersurface case such a field extension has been studied by Pushnikov [P90a, P90b].) The transcendence degree $m_{p}(f)$ of the above field extension is called the transcendence degree of the CR-map $f$ at $p$ (see [CMS99, CPS00]). We have the following standard relation between $m_{p}(f)$ and $\mu_{p}(f)$ :

LEMMA 4.2. Let $M \subset \mathbb{C}^{N}$ be a generic real-analytic submanifold through some point $p \in M$ and $f: M \rightarrow \mathbb{C}^{N^{\prime}} a \mathcal{C}^{\infty}$-smooth $C R$-map. Assume that $M$ is minimal at p. Then $\mu_{p}(f)=N+m_{p}(f)$. 
REMARK 4.3. The minimality of $M$ is needed to guarantee that $\mathcal{M}_{p}(M)\left(f_{1}, \ldots, f_{N^{\prime}}\right)$ is a field so that the transcendence degree is defined.

The following well-known proposition shows the relevance of $\mu_{p}(f)$ for the study of the holomorphic extension of $f$.

Proposition 4.4. Let $M \subset \mathbb{C}^{N}$ be a generic real-analytic submanifold through a point $p$ and $f: M \rightarrow \mathbb{C}^{N^{\prime}} a \mathcal{C}^{\infty}$-smooth CR-map. Then the following are equivalent:

(i) $\mu_{p}(f)=N$;

(ii) $f$ is real-analytic near $p$.

(iii) $f$ extends holomorphically to a neighborhood of $p$ in $\mathbb{C}^{N}$.

Proposition 4.4 is a consequence of theorems of TOMASSINI [To66] and of MALGRANGE [Ma66].

\section{Local geometry of the Zariski closure.}

5.1. Preliminaries. We use the notation from $\S 4$ and assume that $M$ is minimal at $p$ and that

$$
\mu_{p}(f)<N+N^{\prime}
$$

holds. By shrinking $M$ around $p$ if necessary, we may assume that $M$ is connected and minimal at all its points. In what follows, for an open subset $\Omega \subset \mathbb{C}^{k}, \mathcal{O}(\Omega)$ will denote the ring of holomorphic functions in $\Omega$.

In $\S 4$, we saw that $\mu_{p}(f) \geq N$ and $m:=m_{p}(f)=\mu_{p}(f)-N$ coincides with the transcendence degree of the field extension $\mathcal{M}_{p}(M) \subset \mathcal{M}_{p}(M)\left(f_{1}, \ldots, f_{N^{\prime}}\right)$, where $f=\left(f_{1}, \ldots, f_{N^{\prime}}\right)$. This implies that there exist integers $1 \leq j_{1}<\ldots<j_{m}<N^{\prime}$ such that $f_{j_{1}}, \ldots, f_{j_{m}}$ form a transcendence basis of $\mathcal{M}_{p}(M)(f)$ over $\mathcal{M}_{p}(M)$. After renumbering the coordinates $z^{\prime}:=(\zeta, w) \in \mathbb{C}^{m} \times \mathbb{C}^{N^{\prime}-m}$ and setting $m^{\prime}:=N^{\prime}-m$, we may assume that

$$
f=(g, h) \in \mathbb{C}_{\zeta}^{m} \times \mathbb{C}_{w}^{m^{\prime}},
$$

where $g=\left(g_{1}, \ldots, g_{m}\right)$ forms a transcendence basis of $\mathcal{M}_{p}(M)(f)$ over $\mathcal{M}_{p}(M)$.

Since the components of the germ at $p$ of the CR-map $h: M \rightarrow \mathbb{C}^{m^{\prime}}$ are algebraically dependent over $\mathcal{M}_{p}(M)(g)$, there exist monic polynomials $P_{j}(T) \in$ $\mathcal{M}_{p}(M)(g)[T], j=1, \ldots, m^{\prime}$, such that if $h=\left(h_{1}, \ldots, h_{m^{\prime}}\right)$, then

$$
P_{j}\left(h_{j}\right)=0, j=1, \ldots, m^{\prime}, \text { in } \mathcal{M}_{p}(M)(f) .
$$

As a consequence, there exist non-trivial polynomials $\widehat{P_{j}}(T) \in \mathcal{O}_{p}(M)[g][T], j=$ $1, \ldots, m^{\prime}$, such that

$$
\widehat{P_{j}}\left(h_{j}\right)=0, j=1, \ldots, m^{\prime} .
$$

For every $j=1, \ldots, m^{\prime}$, we can write

$$
\widehat{P_{j}}(T)=\sum_{\nu \leq k_{j}} q_{j \nu} T^{\nu}
$$

where each $q_{j \nu} \in \mathcal{O}_{p}(M)[g], q_{j k_{j}} \not \equiv 0$ and $k_{j} \geq 1$. Since each $q_{j \nu}$ is in $\mathcal{O}_{p}(M)[g]$, we can also write

$$
q_{j \nu}=q_{j \nu}(z)=R_{j \nu}(z, g(z))
$$


where $R_{j \nu}(z, \zeta) \in \mathcal{O}_{p}(M)[\zeta]$. Note that each $R_{j \nu}(z, \zeta)$ can also be viewed as an element of $\mathcal{O}_{p}[\zeta]$.

Let $\Delta_{p}^{N}$ be a polydisc neighborhood of $p$ in $\mathbb{C}^{N}$ such that the analogues of (5.4) - (5.6) hold with germs replaced by their representatives in $M \cap \Delta_{p}^{N}$ (denoted by the same letters). Moreover, in view of Remark 4.1, we may assume that the Zariski closure $\mathcal{Z}_{f}$ can be represented by an irreducible (over the ring $\mathcal{O}_{p}\left[z^{\prime}\right]$ ) closed analytic subset of $\Delta_{p}^{N} \times \mathbb{C}^{N^{\prime}}$ (also denoted by $\mathcal{Z}_{f}$ ). By shrinking $M$ we may also assume that $M$ is contained in $\Delta_{p}^{N}$. Hence we have

$$
\Gamma_{f} \subset \mathcal{Z}_{f} \subset \Delta_{p}^{N} \times \mathbb{C}^{N^{\prime}}
$$

Define

$$
\widetilde{P_{j}}(z, \zeta ; T):=\sum_{\nu=0}^{k_{j}} R_{j \nu}(z, \zeta) T^{\nu} \in \mathcal{O}\left(\Delta_{p}^{N}\right)[\zeta][T], j=1, \ldots, m^{\prime} .
$$

It follows from (5.4) - (5.6) that one has

$$
\widetilde{P_{j}}\left(z, g(z) ; h_{j}(z)\right)=0, z \in M, j=1, \ldots, m^{\prime} .
$$

Here each $R_{j \nu}(z, \zeta) \in \mathcal{O}\left(\Delta_{p}^{N}\right)[\zeta], k_{j} \geq 1$, and

$$
R_{j k_{j}}(z, g(z)) \not \equiv 0, \quad z \in M \text {. }
$$

Moreover, since $\mathcal{O}_{p}[\zeta][T]$ is a unique factorization domain (see e.g. [ZS58]) and since $M$ is minimal at $p$, we may assume that the polynomials given by (5.8) are irreducible. by

Consider the complex-analytic variety $\mathcal{V}_{f} \subset \mathbb{C}^{N} \times \mathbb{C}^{N^{\prime}}$ through $(p, f(p))$ defined

$$
\mathcal{V}_{f}:=\left\{(z, \zeta, w) \in \Delta_{p}^{N} \times \mathbb{C}^{m} \times \mathbb{C}^{m^{\prime}}: \widetilde{P}_{j}\left(z, \zeta ; w_{j}\right)=0, j=1, \ldots, m^{\prime}\right\}
$$

By $(5.9), \mathcal{V}_{f}$ contains the graph $\Gamma_{f}$ and hence the Zariski closure $\mathcal{Z}_{f}$. In fact, since by Lemma $4.2, \operatorname{dim}_{\mathbb{C}} \mathcal{Z}_{f}=\mu_{p}(f)=N+m$, it follows from the construction that $\mathcal{Z}_{f}$ is the (unique) irreducible component of $\mathcal{V}_{f}$ (over $\mathcal{O}_{p}\left[z^{\prime}\right]$ ) containing $\Gamma_{f}$. Note that $\mathcal{V}_{f}$ is not irreducible in general and, moreover, can have a dimension larger than $\mu_{p}(f)$. (This may happen, e.g. if another component of $\mathcal{V}_{f}$ is of higher dimension than $\mu_{p}(f)$ ).

For $j=1, \ldots, m^{\prime}$, let $\widetilde{D_{j}}(z, \zeta) \in \mathcal{O}\left(\Delta_{p}^{N}\right)[\zeta]$ be the discriminant of the polynomial $\widetilde{P_{j}}(z, \zeta ; T)$ (with respect to $T$ ). Consider the complex-analytic set

$$
\widetilde{\mathcal{D}}:=\cup_{j=1}^{m^{\prime}}\left\{(z, \zeta) \in \Delta_{p}^{N} \times \mathbb{C}^{m}: \widetilde{D_{j}}(z, \zeta)=0\right\}
$$

By the irreducibility of each polynomial $\widetilde{P_{j}}(z, \zeta ; T)$, we have $\widetilde{D_{j}}(z, \zeta) \not \equiv 0$ in $\Delta_{p}^{N} \times \mathbb{C}^{m}$, for $j=1, \ldots, m^{\prime}$. Therefore from the algebraic independence of the components of the map $g$ over $\mathcal{M}_{p}(M)$, it follows that the graph of $g$ is not contained in $\widetilde{\mathcal{D}}$, i.e. that for $z \in M$,

$$
\widetilde{D_{j}}(z, g(z)) \not \equiv 0, \text { for } j=1, \ldots, m^{\prime} .
$$

By minimality of $M$ as before, the sets

$$
\Sigma_{j}:=\left\{z \in M: \widetilde{D_{j}}(z, g(z))=0\right\}, j=1, \ldots, m^{\prime},
$$

are nowhere dense in $M$, and so is the set

$$
\Sigma:=\cup_{j=1}^{m^{\prime}} \Sigma_{j}=\{z \in M:(z, g(z)) \in \widetilde{\mathcal{D}}\} .
$$


5.2. Description of $\mathcal{Z}_{f}$ on a dense subset of the graph of $f$. By the implicit function theorem, for any point $z_{0} \in M \backslash \Sigma$, there exist polydisc neighborhoods of $z_{0}, g\left(z_{0}\right)$ and $h\left(z_{0}\right)$, denoted by $\Delta_{z_{0}}^{N} \subset \Delta_{p}^{N} \subset \mathbb{C}^{N}, \Delta_{g\left(z_{0}\right)}^{m} \subset \mathbb{C}^{m}, \Delta_{h\left(z_{0}\right)}^{m^{\prime}} \subset \mathbb{C}^{m^{\prime}}$ respectively and a holomorphic map

$$
\theta\left(z_{0} ; \cdot\right): \Delta_{z_{0}}^{N} \times \Delta_{g\left(z_{0}\right)}^{m} \rightarrow \Delta_{h\left(z_{0}\right)}^{m^{\prime}}
$$

such that for $(z, \zeta, w) \in \Delta_{z_{0}}^{N} \times \Delta_{g\left(z_{0}\right)}^{m} \times \Delta_{h\left(z_{0}\right)}^{m^{\prime}}$,

$$
(z, \zeta, w) \in \mathcal{V}_{f} \Longleftrightarrow(z, \zeta, w) \in \mathcal{Z}_{f} \Longleftrightarrow w=\theta\left(z_{0} ; z, \zeta\right) \text {. }
$$

Since $\Gamma_{f} \subset \mathcal{Z}_{f}$ in view of (5.16), for every fixed $z_{0} \in M \backslash \Sigma$, we have

$$
h(z)=\theta\left(z_{0} ; z, g(z)\right), z \in M \cap \Delta_{z_{0}}^{N} \text {. }
$$

Let $Z_{f} \subset M \times \mathbb{C}^{N^{\prime}}$ be the real-analytic subset given by

$$
Z_{f}:=\mathcal{Z}_{f} \cap\left(M \times \mathbb{C}^{N^{\prime}}\right)
$$

and, for every $z_{0} \in M \backslash \Sigma$, consider the real-analytic submanifold $Z_{f}\left(z_{0}\right) \subset Z_{f}$ defined by setting

$$
Z_{f}\left(z_{0}\right):=Z_{f} \cap\left(\Delta_{z_{0}}^{N} \times \Delta_{g\left(z_{0}\right)}^{m} \times \Delta_{h\left(z_{0}\right)}^{m^{\prime}}\right)
$$

Note that $Z_{f}\left(z_{0}\right)$ contains the graph of $f$ over $M \cap \Delta_{z_{0}}^{N}$ and that, by making the holomorphic change of coordinates $\left(\widetilde{z}, \widetilde{z}^{\prime}\right)=\left(z, \varphi\left(z, z^{\prime}\right)\right) \in \mathbb{C}^{N} \times \mathbb{C}^{N^{\prime}}$ where $\varphi\left(z, z^{\prime}\right)=\varphi(z,(\zeta, w)):=\left(\zeta, w-\theta\left(z_{0} ; z, \zeta\right)\right)$, the submanifold $Z_{f}\left(z_{0}\right)$ is given in these new coordinates by.

$$
Z_{f}\left(z_{0}\right)=\left\{\left(\widetilde{z}, \widetilde{z}^{\prime}\right) \in \Delta_{z_{0}}^{N} \times \Delta_{g\left(z_{0}\right)}^{m} \times \mathbb{C}^{m^{\prime}}: \widetilde{z} \in M, \widetilde{z}_{m+1}^{\prime}=\ldots=\widetilde{z}_{N^{\prime}}^{\prime}=0\right\},
$$

where we write $\widetilde{z}^{\prime}=\left(\widetilde{z}_{1}^{\prime}, \ldots, \widetilde{z}_{N^{\prime}}^{\prime}\right)$.

We summarize the above in the following proposition.

Proposition 5.1. Let $M \subset \mathbb{C}^{N}$ be a generic real-analytic submanifold through a point $p \in M$ and $f: M \rightarrow \mathbb{C}^{N^{\prime}}$ a $\mathcal{C}^{\infty}$-smooth $C R$-map. Let $\mathcal{Z}_{f}$ be the local Zariski closure at $(p, f(p))$ of the graph of $f$ as defined in $\S 4.1$. Assume that $M$ is minimal at $p$ and that $\mu_{p}(f)<N+N^{\prime}$. Then after shrinking $M$ around $p$, the following holds. For $z_{0} \in M \backslash \Sigma$, where $\Sigma$ is the nowhere dense open subset of $M$ given by (5.14), there exists a holomorphic change of coordinates near $\left(z_{0}, f\left(z_{0}\right)\right)$ of the form $\left(\widetilde{z}, \widetilde{z}^{\prime}\right)=\left(z, \varphi\left(z, z^{\prime}\right)\right) \in \mathbb{C}^{N} \times \mathbb{C}^{N^{\prime}}$ such that the real-analytic subset $\mathcal{Z}_{f} \cap\left(M \times \mathbb{C}^{N^{\prime}}\right)$ is given near $\left(z_{0}, f\left(z_{0}\right)\right)$ by $(5.20)$, with $m=\mu_{p}(f)-N$.

For every $z_{0} \in M \backslash \Sigma$, denote by $\Omega_{z_{0}}$ the (unique) connected component of $\left(M \cap \Delta_{z_{0}}^{N}\right) \times \Delta_{g\left(z_{0}\right)}^{m}$ passing through $\left(z_{0}, g\left(z_{0}\right)\right)$. Since $\Omega_{z_{0}}$ is connected, it makes sense to consider the quotient field of the ring of real-analytic functions $\mathcal{C}^{\omega}\left(\Omega_{z_{0}}\right)$ that we denote by $\mathcal{K}\left(z_{0}\right)$. Let

$$
j: \mathcal{C}^{\omega}(M)[\zeta, \bar{\zeta}] \rightarrow \mathcal{C}^{\omega}\left(\Omega_{z_{0}}\right)
$$

be the restriction map and

$$
\mathcal{D}:=\operatorname{Im} j \subset \mathcal{C}^{\omega}\left(\Omega_{z_{0}}\right)
$$


be the image of $j$. Note that, since $\Omega_{z_{0}}$ is open in $M \times \mathbb{C}^{m}, j$ is an injective ring homomorphism and hence, one can identify $\mathcal{D}$ with $\mathcal{C}^{\omega}(M)[\zeta, \bar{\zeta}]$ via $j$. Denote by $\mathcal{F}$ the quotient field of $\mathcal{D}$. The field $\mathcal{F}$ is naturally identified with the field of all rational functions in $(\zeta, \bar{\zeta})$ with coefficients that extend as real-analytic functions on $M$. We have the field extension $\mathcal{F} \subset \mathcal{K}\left(z_{0}\right)$.

The following lemma, which will be needed for the proof of Theorem 1.8, is a direct consequence of (5.16) (see also [CMS99] for a related argument):

Lemma 5.2. For every fixed $z_{0} \in M \backslash \Sigma$, the restriction of the map $\theta\left(z_{0} ; z, \zeta\right)$ (given by (5.16)) to $\Omega_{z_{0}}$ satisfies a nontrivial polynomial identity with coefficients in $\mathcal{O}\left(\Delta_{p}^{N}\right)[\zeta]$

6. Proof of Theorem 1.8. With all the tools defined in $\S 4-\S 5$ at our disposal, we are now ready to prove the following statement from which Theorem 1.8 will follow.

THEOREM 6.1. Let $M \subset \mathbb{C}^{N}$ be a real-analytic generic submanifold through a point $p \in M$. Let $f: M \rightarrow \mathbb{C}^{N^{\prime}}$ be a $\mathcal{C}^{\infty}$-smooth $C R$-map and $\mathcal{Z}_{f}$ the local Zariski closure over $\mathcal{O}_{p}\left[z^{\prime}\right]$ at $(p, f(p))$ of $\Gamma_{f}$ as defined in $\S 4.1$. Suppose that $M$ is minimal at $p$ and $f$ maps $M$ into $M^{\prime}$, where $M^{\prime}$ is a proper real-algebraic subset of $\mathbb{C}^{N^{\prime}}$. Then, shrinking $M$ around $p$ and choosing an appropriate union $\widetilde{Z}_{f}$ of local realanalytic irreducible components of $\mathcal{Z}_{f} \cap\left(M \times \mathbb{C}^{N^{\prime}}\right)$ at $(p, f(p))$ if necessary, one has the following:

(i) $\mu_{p}(f)<N+N^{\prime}$ for $\mu_{p}(f)=\operatorname{dim} \mathcal{Z}_{f}$;

(ii) $\Gamma_{f} \subset \widetilde{Z}_{f} \subset M \times M^{\prime}$;

(iii) $\widetilde{Z}_{f}$ satisfies the following straightening property: for any point $q$ in a dense subset of $M$, there exists a neighborhood $U_{q}$ of $(q, f(q))$ in $\mathbb{C}^{N} \times \mathbb{C}^{N^{\prime}}$ and a holomorphic change of coordinates in $U_{q}$ of the form $\left(\widetilde{z}, \widetilde{z}^{\prime}\right)=\Phi\left(z, z^{\prime}\right)=$ $\left(z, \varphi\left(z, z^{\prime}\right)\right) \in \mathbb{C}^{N} \times \mathbb{C}^{N^{\prime}}$ such that

$$
\widetilde{Z}_{f} \cap U_{q}=\left\{\left(z, z^{\prime}\right) \in U_{q}: z \in M, \widetilde{z}_{m+1}^{\prime}=\cdots=\widetilde{z}_{N^{\prime}}^{\prime}=0\right\},
$$

where $m=\mu_{p}(f)-N$.

For the proof we shall need the following result.

Proposition 6.2. Under the assumptions of Theorem 6.1, shrinking $M$ around $p$ if necessary, one has the following:

(i) $\mu_{p}(f)<N+N^{\prime}$;

(ii) For any point $z_{0} \in M \backslash \Sigma$, the real-analytic submanifold $Z_{f}\left(z_{0}\right)$ is contained in $M \times M^{\prime}$, where $\Sigma$ is the nowhere dense subset of $M$ given by (5.14) and $Z_{f}\left(z_{0}\right) \subset \mathcal{Z}_{f} \cap\left(M \times \mathbb{C}^{N^{\prime}}\right)$ is given by (5.19).

Proof. [Proof of Proposition 6.2 (i)] We proceed by contradiction. Suppose that the dimension $\mu_{p}(f)$ of the local Zariski closure is $N+N^{\prime}$. Since $M^{\prime}$ is a proper real-algebraic subset of $\mathbb{C}^{N^{\prime}}$, there exists a nontrivial polynomial $\rho^{\prime}\left(z^{\prime}, \overline{z^{\prime}}\right) \in \mathbb{C}\left[z^{\prime}, \overline{z^{\prime}}\right]$ vanishing on $M^{\prime}$. Since $f$ maps $M$ into $M^{\prime}$, we have

$$
\rho^{\prime}(f(z), \overline{f(z)})=0
$$

for all $z \in M$. It follows from Proposition 3.4 (ii) (applied to $F:=f=\left(f_{1}, \ldots, f_{N^{\prime}}\right)$ ) that the germs at $p$ of the components $f_{1}, \ldots, f_{N^{\prime}}$ satisfy a nontrivial polynomial identity with coefficients in $\mathcal{O}_{p}(M)$. This contradicts the assumption $\mu_{p}(f)=N+N^{\prime}$. The proof of Proposition 6.2 (i) is complete. 
In view of Proposition 6.2 (i), we may now assume to be in the setting of $\S 4-\S 5$. Since $M^{\prime}$ is real-algebraic, it is given by

$$
M^{\prime}:=\left\{z^{\prime} \in \mathbb{C}^{N^{\prime}}: \rho_{1}^{\prime}\left(z^{\prime}, \overline{z^{\prime}}\right)=\ldots=\rho_{l}^{\prime}\left(z^{\prime}, \overline{z^{\prime}}\right)=0\right\},
$$

where each $\rho_{j}^{\prime}\left(z^{\prime}, \overline{z^{\prime}}\right)$, for $j=1, \ldots, l$, is a real-valued polynomial in $\mathbb{C}\left[z^{\prime}, \overline{z^{\prime}}\right]$. For $j=1, \ldots, l, z_{0} \in M \backslash \Sigma$ and $(z, \zeta) \in \Omega_{z_{0}}$, define

$$
r_{j}(z, \bar{z}, \zeta, \bar{\zeta}):=\rho_{j}^{\prime}\left(\zeta, \theta\left(z_{0} ; z, \zeta\right), \bar{\zeta}, \overline{\theta\left(z_{0} ; z, \zeta\right)}\right) \in \mathcal{C}^{\omega}\left(\Omega_{z_{0}}\right)
$$

where $\theta\left(z_{0} ; \cdot\right): \Omega_{z_{0}} \rightarrow \Delta_{h\left(z_{0}\right)}^{m^{\prime}}$ is the restriction to $\Omega_{z_{0}}$ of the holomorphic map given by (5.16) and $\Omega_{z_{0}}$ is the open subset of $M \times \mathbb{C}^{m}$ given in $\S 5.2$. We need the following lemma.

LEMMA 6.3. For every $z_{0} \in M \backslash \Sigma$ and $j=1, \ldots$, , the real-analytic function $r_{j}$ satisfies a nontrivial polynomial identity on $\Omega_{z_{0}}$ with coefficients in $\mathcal{C}^{\omega}(M)[\zeta, \bar{\zeta}]$.

Proof. It follows from Lemma 5.2 that each component of the restriction to $\Omega_{z_{0}}$ of $\theta\left(z_{0} ; \cdot\right)$, considered as an element of $\mathcal{C}^{\omega}\left(\Omega_{z_{0}}\right)$, is algebraic over the field $\mathcal{F}$ defined in $\S 5.2$. Therefore, in view of the definition of $\mathcal{F}$, it is also the case for each component of the restriction to $\Omega_{z_{0}}$ of $\overline{\theta\left(z_{0} ; \cdot\right)}$. Since for $j=1, \ldots, l$, each $\rho_{j}^{\prime}\left(z^{\prime}, \overline{z^{\prime}}\right)$ is a polynomial, it follows from (6.4) that each $r_{j}$ belongs to the field generated by $\mathcal{F}$ and the components of the restriction to $\Omega_{z_{0}}$ of the maps $\theta\left(z_{0} ; \cdot\right)$ and $\overline{\theta\left(z_{0} ; \cdot\right)}$. Hence, by standard arguments from field theory (see e.g. [ZS58]), each $r_{j}$ is also algebraic over $\mathcal{F}$ for $j=1, \ldots, l$, which gives the desired statement of the lemma.

Proof. [Proof of Proposition 6.2 (ii).] By contradiction, assume that there exists $z_{0} \in M \backslash \Sigma$ such that the real-analytic submanifold $Z_{f}\left(z_{0}\right)$ given by (5.19) is not contained in $M \times M^{\prime}$. In view of (5.16), (5.18), (5.19) and (6.4), this means that there exists $j_{0} \in\{1, \ldots, l\}$ such that $r_{j_{0}} \not \equiv 0$ in $\Omega_{z_{0}}$. By Lemma 6.3, there exists a nontrivial polynomial $Q(z, \bar{z}, \zeta, \bar{\zeta} ; T) \in \mathcal{C}^{\omega}(M)[\zeta, \bar{\zeta}][T]$ such that

$$
Q\left(z, \bar{z}, \zeta, \bar{\zeta} ; r_{j_{0}}(z, \bar{z}, \zeta, \bar{\zeta})\right) \equiv 0, \quad \text { for }(z, \zeta) \in \Omega_{z_{0}}
$$

Moreover, since $r_{j_{0}}$ does not vanish identically on $\Omega_{z_{0}}$ and $M$ is connected, we may choose $Q$ such that

$$
Q(z, \bar{z}, \zeta, \bar{\zeta} ; 0) \not \equiv 0 \text { for }(z, \zeta) \in M \times \mathbb{C}^{m}
$$

Recall that we write $f=(g, h)$ as in (5.2) and that the graph of $g=\left(g_{1}, \ldots, g_{m}\right)$ over $M \cap \Delta_{z_{0}}^{N}$ is contained in $\Omega_{z_{0}}$. Then (6.5) implies that for $z \in M \cap \Delta_{z_{0}}^{N}$,

$$
Q\left(z, \bar{z}, g(z), \overline{g(z)} ; r_{j_{0}}(z, \bar{z}, g(z), \overline{g(z)})\right) \equiv 0 .
$$

But since $f$ maps $M$ into $M^{\prime}$, we have for $j=1, \ldots, l$,

$$
\rho_{j}^{\prime}(f(z), \overline{f(z)}) \equiv \rho_{j}^{\prime}(g(z), h(z), \overline{g(z)}, \overline{h(z)}) \equiv 0, \quad z \in M .
$$

Therefore, in view of (5.17), (6.4) and (6.8), we obtain that for all $z \in M \cap \Delta_{z_{0}}^{N}$,

$$
r_{j_{0}}(z, \bar{z}, g(z), \overline{g(z)}) \equiv 0 .
$$

From (6.7) and (6.9), we conclude that for all $z \in M \cap \Delta_{z_{0}}^{N}$,

$$
Q(z, \bar{z}, g(z), \overline{g(z)} ; 0) \equiv 0
$$


In view of (6.6), condition (ii) in Proposition 3.4 is satisfied for the components $g_{1}, \ldots, g_{m}$ of $g$ that are $\mathcal{C}^{\infty}$-smooth CR-functions on $M$. By Proposition 3.4, the germs at $p$ of $g_{1}, \ldots, g_{m}$ satisfy a nontrivial polynomial identity with coefficients in $\mathcal{O}_{p}(M)$. This contradicts the fact that $g_{1}, \ldots, g_{m}$ form a transcendence basis of $\mathcal{M}_{p}(M)(f)$ over $\mathcal{M}_{p}(M)$ (see $\S 5.1$ ). The proof is complete.

Proof. [Proof of Theorem 6.1] We shrink $M$ so that the conclusion of Proposition 6.2 holds. Define $\widetilde{Z}_{f}$ to be the union of those irreducible real-analytic components of $\mathcal{Z}_{f} \cap\left(M \times \mathbb{C}^{N^{\prime}}\right)$ that contain open pieces of $\Gamma_{f}$. Then the conclusions (i) and (ii) of Theorem 6.1 follow from Proposition 6.2 and the straightening property (iii) follows from Proposition 5.1.

Proof. [Proof of Theorem 1.8] Without loss of generality, we may assume that $M$ is generic. Since $f$ does not extend holomorphically to any neighborhood of $p$ in $\mathbb{C}^{N}$, we have $n:=\mu_{p}(f)-N>0$ by Proposition 4.4. Then Theorem 1.8 follows immediately from Theorem 6.1.

\section{Proofs of Theorems 1.1, 1.3, 1.6, 1.7.}

Proof. [Proof of Theorem 1.1] We need to prove that if $f$ does not extend holomorphically to any neighborhood of $p$ in $\mathbb{C}^{N}$, then necessarily $f$ maps a neighborhood of $p$ in $M$ into $\mathcal{E}^{\prime}$. By Theorem 1.8, there exists a neighborhood $U$ of $p$ in $M$ such that for all points $q$ in a dense open subset of $U$, one has $f(q) \in \mathcal{E}^{\prime}$. Since the set $\mathcal{E}^{\prime}$ is closed in $M^{\prime}$ (see $\S 1$ ), it follows that $f(U) \subset \mathcal{E}^{\prime}$. This completes the proof of Theorem 1.1 .

Proof. [Proof of Theorem 1.3] We may assume that $M$ is generic. Since $M$ is real-algebraic, connected and minimal somewhere, it is minimal outside a proper real-algebraic subset $S$. In view of Corollary 1.2, we may assume that $p \in S$.

If $W$ is a connected component of $M \backslash S$, then we claim that either $f$ is realalgebraic on $W$ or $f(W) \subset V^{\prime}$. Indeed, if $f(W) \not \subset V^{\prime}$, then $f$ extends holomorphically to a neighborhood in $\mathbb{C}^{N}$ of some point $q \in W$ by Corollary 1.2. Therefore, it is realalgebraic by a result of the third author [Z99], i.e. every component of $f$ satisfies a nontrivial polynomial identity in a neighborhood of $q$ in $M$. Then by Tumanov's theorem and unique continuation, it follows that the same polynomial identities for the components of $f$ hold everywhere on $W$ and hence $f$ is real-algebraic on $W$.

By repeating the arguments from $[\mathrm{DF} 78, \S 6]$ one can show that, near $p^{\prime}:=f(p)$, the set $V^{\prime}$ (which may be empty) is complex-algebraic, i.e. given by the vanishing of a vector-valued holomorphic polynomial $P\left(z^{\prime}\right), z^{\prime} \in \mathbb{C}^{N^{\prime}}$. Then, by the above claim, $P \circ f$ is real-algebraic on each connected component of $M \backslash S$. It is known (see e.g. [BR90]) that some neighborhood of $p$ in $M$ intersects only finitely many connected components of $M \backslash S$. Hence $P \circ f$ is real-algebraic in a neighborhood of $p$ in $M$ and therefore, since $P \circ f$ is $\mathcal{C}^{\infty}$-smooth, it is real-analytic by MALGRANGE's theorem (see [Ma66]).

If $f$ does not send a connected neighborhood of $p$ in $M$ into $V^{\prime}$, the real-analytic map $P \circ f$ does not vanish identically on each of the components of $M \backslash S$ intersecting this neighborhood. Hence, by the above claim, $f$ is real-algebraic on each such component and therefore in a neighborhood of $p$. Then the required holomorphic extension of $f$ at $p$ follows from MALGRANGE's and TOMASSINI's theorems.

For the proof of Theorem 1.6, it will be convenient to derive the following corollary from Theorem 1.8 . 
CoROLlaRY 7.1. Under the assumptions of Theorem 1.8 suppose furthermore that $M$ and $M^{\prime}$ have the same $C R$-codimension and $f$ is submersive at points arbitrarily close to $p$. Then there exists an integer $1 \leq n \leq N^{\prime}-1$ and a point $q^{\prime} \in M^{\prime}$ arbitrarily close to $f(p)$ such that an open neighborhood of $q^{\prime}$ in $M^{\prime}$ is biholomorphically equivalent to a product $Y \times \omega$, where $Y \subset \mathbb{C}^{N^{\prime}-n}$ is a real-analytic submanifold and $\omega$ is an open subset in $\mathbb{C}^{n}$.

Proof. [Proof of Corollary 7.1] Let $A, n$ be given by Theorem 1.8, $\pi^{\prime}: \mathbb{C}_{z}^{N} \times \mathbb{C}_{z^{\prime}}^{N^{\prime}} \rightarrow$ $\mathbb{C}_{z^{\prime}}^{N^{\prime}}$ the natural projection and fix a sufficiently small open neighborhood $U^{\prime}$ of $f(p)$ in $\mathbb{C}^{N^{\prime}}$. Choose a point $q \in M$ such that $f$ is submersive at $q$ and $f(q) \in U^{\prime}$. Let $U_{q}, \varphi$ be as in Theorem 1.8 and set $\Phi\left(z, z^{\prime}\right):=\left(z, \varphi\left(z, z^{\prime}\right)\right)$. Shrinking $U_{q}$ if necessary, we may assume that $\pi^{\prime}\left(A \cap U_{q}\right) \subset U^{\prime}$ and $\left.\pi^{\prime}\right|_{A \cap U_{q}}: A \cap U_{q} \rightarrow M^{\prime} \cap U^{\prime}$ is submersive. By the conclusion of Theorem 1.8, there exists a neighborhood $M_{q} \subset M$ of $q$ and an open subset $\omega \subset \mathbb{C}^{n}$ such that $\Phi\left(A \cap U_{q}\right)=M_{q} \times \omega \times\{0\}$. Since $\left.\pi^{\prime}\right|_{A \cap U_{q}}$ is a submersion on $M^{\prime}$, its extension $\pi^{\prime} \circ \Phi^{-1}$ defines a holomorphic submersion between intrinsic complexifications $\mathcal{A}$ and $\mathcal{M}^{\prime}$ of $A$ and $M^{\prime}$ respectively near corresponding points. In particular, there exists a complex submanifold $V \subset \mathbb{C}^{N}$ through $q$ such that $\psi:=\left.\pi^{\prime} \circ \Phi^{-1}\right|_{V \times \omega \times\{0\}}$ is biholomorphic onto $\mathcal{M}^{\prime}$. From the equality of the CR-codimensions of $M$ and $M^{\prime}$, it follows that $Y:=M \cap V$ is a CR-submanifold of $\mathbb{C}^{N}$ and that the biholomorphism $\psi$ sends $Y \times \omega \times\{0\}$ onto a neighborhood of $f(q)$ in $M^{\prime}$. The proof is complete.

Proof. [Proof of Theorem 1.6] Let $M, M^{\prime}$ and $f$ be as in Theorem 1.6. Then $f$ is submersive at a point $p_{0} \in M$. If $f$ were not holomorphically extendible to a neighborhood of $p_{0}$ in $\mathbb{C}^{N}$, there would exist an open holomorphically degenerate submanifold $Y^{\prime} \subset M^{\prime}$ by Corollary 7.1. This would contradict the assumption that $M^{\prime}$ is holomorphically nondegenerate. Hence $f$ is real-analytic at $p_{0}$. Now define $\Omega \subset M$ to be the maximal connected open subset containing $p_{0}$ where $f$ is real-analytic. Then $f$ is submersive on a dense subset of $\Omega$. We claim that $\Omega=M$. Otherwise there would exist $p \in \bar{\Omega}$ where $f$ is not real-analytic that would contradict Corollary 7.1 as before. Hence $f$ is real-analytic everywhere on $M$ and the proof is complete.

Proof. [Proof of Theorem 1.7] As in the proof of Theorem 1.3, we may assume that $M$ is generic and we let $S \subset M$ be the real-algebraic subset of all nonminimal points. By assumption, $f$ is submersive at a point $p_{0} \in M$ which can be assumed minimal without loss of generality. By Theorem 1.6, $f$ extends holomorphically to a neighborhood in $\mathbb{C}^{N}$ of the connected component $W_{0}$ of $p_{0}$ in $M \backslash S$. Since both $M$ and $M^{\prime}$ are real-algebraic, $f$ is real-algebraic on $W_{0}$ by a result of [Z99]. The same argument shows that, for every connected component $W$ of $M \backslash S$, either $f$ is real-algebraic or it is nowhere submersive on $W$.

As in the proof of Theorem 1.6, define $\Omega \subset M$ to be the maximal open connected subset containing $p_{0}$ where $f$ is real-analytic. Then $f$ is submersive on a dense subset of $\Omega$. Assume by contradiction that $f$ is not real-analytic everywhere on $M$ and hence that there exists a point $p_{1} \in \bar{\Omega}$ where $f$ is not real-analytic. Fix local real-algebraic coordinates in $M$ and $M^{\prime}$ near $p_{1}$ and $f\left(p_{1}\right)$ respectively and denote by $\Delta$ a minor of the Jacobian matrix of $f$ of the maximal size that does not vanish identically in any neighborhood of $p_{1}$. By the first part of the proof, we conclude that $\Delta$ is real-algebraic and hence real-analytic in a connected neighborhood $U$ of $p_{1}$ in $M$. In particular, $f$ is submersive on a dense subset of $U$. Hence $f$ is real-algebraic on every component of $U \backslash S$ by the first part of the proof again, and hence, by MALGRANGE's theorem, it follows that $f$ is real-analytic near $p_{1}$, which is a contradiction. This shows that 
$\Omega=M$ and hence concludes the proof of the theorem.

\section{REFERENCES}

[BER96] Baouendi, M.S.; Ebenfelt, P.; Rothschild, L.P., Algebraicity of holomorphic mappings between real algebraic sets in $\mathbb{C}^{n}$, Acta Math., 177 (1996), pp. 225-273.

[BER99] BAouendi, M.S.; Ebenfelt, P.; Rothschild, L.P., Real Submanifolds in Complex Space and Their Mappings, Princeton Math. Series, 47, Princeton Univ. Press, 1999.

[BHR96] Baouendi, M.S.; Huang, X.; Rothschild L.P., Regularity of CR mappings between algebraic hypersurfaces, Invent. math., 125 (1996), pp. 13-36.

[BR90] Benedetti, R.; Risler, J.-J., Real algebraic and semi-algebraic sets, Actualites Mathematiques. Hermann Editeurs des Sciences et des Arts, 1990.

[CMS99] Coupet, B.; Meylan, F.; Sukhov, A., Holomorphic maps of algebraic CR manifolds, Int. Math. Res. Not., 1 (1999), pp. 1-29.

[CPS99] Coupet, B.; Pinchuk, S.; Sukhov, A., Analyticité des applications CR, C. R. Acad. Sci. Paris Sér. I Math., 329:6 (1999), pp. 489-494.

[CPSO0] Coupet, B.; Pinchuk, S.; Sukhov, A., On partial analyticity of $C R$ mappings, Math. Z., 235 (2000), pp. 541-557.

[D'A82] D'Angelo, J.P., Real hypersurfaces, orders of contact, and applications, Ann. of Math. (2), 115:3 (1982), pp. 615-637.

[D'A91] D'Angelo, J.P., Finite type and the intersection of real and complex subvarieties, Several complex variables and complex geometry, Part 3 (Santa Cruz, CA, 1989), pp. 103-117, Proc. Sympos. Pure Math., 52, Part 3, Amer. Math. Soc., Providence, RI, 1991.

[Da01] DAmour, S., On the analyticity of smooth CR mappings between real-analytic CR manifolds, Michigan Math. J., 49:3 (2001), pp. 583-603.

[DF78] Diederich, K.; Forness, J.E., Pseudoconvex domains with real-analytic boundary, Ann. of Math. (2), 107:2 (1978), pp. 371-384.

[E96] EBENFELT, P., On the unique continuation problem for $C R$ mappings into nonminimal hypersurfaces, J. Geom. Anal., 6 (1996), pp. 385-405.

[F86] Forstnerič, F., Proper holomorphic maps from balls, Duke Math. J., 53 (1986), pp. $427-441$.

[F89] ForSTNERIČ, F., Extending proper holomorphic mappings of positive codimension, Invent. Math., 95 (1989), pp. 31-62.

[F92] Forstnerič, F., Mappings of quadric Cauchy-Riemann manifolds, Math. Ann., 292 (1992), pp. 163-180.

[F93] Forstnerič, F., Proper holomorphic mappings: a survey, Several complex variables (Stockholm, 1987/1988), pp. 297-363, Math. Notes, 38, Princeton Univ. Press, Princeton, NJ, 1993.

[H94] HuANG, X., On the mapping problem for algebraic real hypersurfaces in the complex spaces of different dimensions, Ann. Inst. Fourier (Grenoble), 44 (1994), pp. 433463.

[H01] HUANG, X., On some problems in several complex variables and CR geometry, First International Congress of Chinese Mathematicians (Beijing, 1998), pp. 383-396, AMS/IP Stud. Adv. Math., 20, Amer. Math. Soc., Providence, RI, 2001.

[K00] KoJCINOVIC, S., Extension of CR mappings between generic algebraic submanifolds, Ph.D. thesis, UCSD, 2000.

[L86] LEMPERT, L., On the boundary behavior of holomorphic mappings, Contributions to several complex variables, pp. 193-215, Aspects Math., E9, Viehweg, Braunschweig, 1986.

[Ma66] Malgrange, B., Ideals of differentiable functions, Tata Institute of Fundamental Research, Bombay, Oxford University Press, 1966.

[Mi03] Mir, N., Analytic regularity of CR maps into spheres, Math. Res. Lett., 10:4 (2003), pp. $447-457$.

[MMZ02] Meylan, F.; Mir, N.; Zaitsev, D., Analytic regularity of CR-mappings, Math. Res. Lett., 9:1 (2002), pp. 71-91.

[P90a] Pushnikov, A.Yu., Holomorphicity of CR-mappings into a space of large dimension, Mat. Zametki, 48:3 (1990), pp. 147-149.

[P90b] Pushnikov, A.Yu., On the holomorphy of CR-mappings of real analytic hypersurfaces, Complex analysis and differential equations, pp. 76-84, Bashkir. Gos. Univ., Ufa, 1990 . 
[S96] Stanton, N., Infinitesimal CR automorphisms of real hypersurfaces, Amer. J. Math., 118 (1996), pp. 209-233.

[To66] Tomassini, G., Tracce delle functional olomorfe sulle sotto varieta analitiche reali d'una varieta complessa, Ann. Scuola Norm. Sup. Pisa, 20 (1966), pp. 31-43.

[Tu88] Tumanov, A.E., Extension of CR-functions into a wedge from a manifold of finite type (Russian), Mat. Sb. (N.S.), 136 (178) (1988), no. 1, pp. 128-139; translation in Math. USSR-Sb., 64:1 (1989), pp. 129-140.

[W79] Webster, S.M., On mapping an $n$-ball into $(n+1)$-ball in complex space, Pac. J. Math., 81 (1979), pp. 267-272.

[Z99] ZAITSEV, D., Algebraicity of local holomorphisms between real-algebraic submanifolds of complex spaces, Acta Math., 183 (1999), pp. 273-305.

[ZS58] Zariski, O.; SAmuel, P., Commutative algebra, Springer-Verlag, Volume 1, 1958. 
